International Journal of Engineering \&Technology, $7(3.12)(2018)$ 175-177
International Journal of Engineering \& Technology
Website: www.sciencepubco.com/index.php/IJET
Research paper

\title{
Fusion Imaging in Pixel Level Image Processing Technique - A Literature Review
}

\author{
K.Elaiyaraja ${ }^{1}$, M.Senthil Kumar ${ }^{2}$ \\ ${ }^{1}$ Assistant Professor-Sr.G/IT, Valliammai Engineering College \\ ${ }^{2}$ Associate Professor/CSE, Valliammai Engineering College \\ *Corresponding Author Email: ${ }^{1}$ k.elaiyaraja@ rediffmail.com
}

\begin{abstract}
Image Processing is an art to get an enriched image or it can be used to retrieve information. This image processing methods are used in medical field also. Numerous modalities like Magnetic Resonance Imaging (MRI), Positron Emission Tomography (PET), and Computed Tomography (CT) etc. are used to analyze and diagnose diseases.Pixel-level image fusion is a combination of several images collected from various inputs and gives more information than any other input messages. Pixel-level image fusion shows a vital role in medical imaging. In this paper, pixel-level image fusionsmethods are survived and review the fusion quality measures are being used. Finally this surveycomplete with different kinds of image fusion methods proposed and still there are so many imminent ways in image fusion applications. Hence image fusions fields are pointedly develop in the forthcoming years.
\end{abstract}

Keywords: Fusion Image, Medical Image Processing, Medical Image Review, Pixel Level, Pixel level image processing.

\section{Introduction}

Image fusion is merging more number of images from different modalities. There are three levels of image fusions. They are Pixel, Feature and Decision level. Pixel-level image fusion merged with the original information of the source images. It produces a fused image which is more informative for photographic observation and computer processing. In a scene more than one image is analyzed to achieve image fusion. For example, images from numerous modalities are merged together to get exact and reliable medical findings. Pixel-level image fusion methods are not only used in medical imaging, also used in remote sensing and Computer vision fields.

\section{Pixel-Level Image Fusion Techniques}

Based on existing image fusion methods, four major classifications can be obtained.

$\checkmark \quad$ Multi-scale decomposition based methods[3]

$\checkmark \quad$ the spars-representation based methods[1,2]

$\checkmark$ Transform domains (Eg. principal component space or the intensity-hue-saturation color space)

$\checkmark$ Hybrid transforms.

Fusion strategy is another significant feature affects fusion outcomes. Fusion strategy is obtained from the coefficients or pixels of the source images.

\section{(a) Multi-Scale Decomposition Based Methods}

The pyramid (such as Laplacian pyramid) and wavelet transform methods (such as Stationary wavelet decomposition, discrete wavelet decomposition etc.) are used commonly in this method. Shift variance, aliasing and lacking in directionality etc. affects discrete wavelet transform[4,5]. The dual-tree complex wavelet transform is used well to avoid those problems[6]. But the edges and curves representations are not good in wavelet schemes. Novel multi-scale geometric tools like curvelet transform[7], contourlet transform are used for the representation of spatial structures[8]. Points discontinuities are detected by using Laplacian pyramid in the contourlet transform[9]. To create bond between point discontinuities and linear structures, Directional filter bank is used. Spatial structures are effectively represented in contourlet transform. So this transform is applied magnificently in medical imaging [10]. No shift-invariant property is caused by downsampling process. The best remedy for this problem is nonsubsampled which consumes more time. Shearlet method applied successfully in image fusion. It is more efficient in computations than contourlet. Edge-preserving filtering also comes in usage for images to represent multi-scale. The quality of fused images are depends up on the number of decomposition levels determined by the multi-scale decomposition method[11].

\section{(b) Sparse Representation Based Methods}

Image processing problems like denoising, interpolation; recognition, etc. are handled properly by using sparse representation. Atoms are collected from the over-complete dictionary[2]. The group of sparse linear atoms describes the image patches and this is how the sparse representation designates the images. Images from multiple sources are segregated into many overlapped patches[12]. So that can obtain the salient features and can preserve shift invariance. These patches then decomposed on the same over-complete dictionary in order to get sparse coefficients. 
Then apply fusion process like choose-max on these coefficients. At the end, the reconstructed images are obtained by the fused coefficients and dictionary.

The following challenges are faced in this sparse representation fusion framework.

$\checkmark \quad$ Sparse code for obtaining coefficients and

$\checkmark$ Dictionary construction.

Orthogonal Matching Pursuit (OMP) algorithm, Gradient sparsity constraint which reflect the sharp edges[13], Simultaneous Orthogonal Matching Pursuit (SOMP) [13] and joint sparsity model are some of the solutions of sparse code for obtaining coefficients.

Next biggest challenge is Dictionary construction[2]. There are two ways used to construct this dictionary. One is the mathematical model based and another one is the example learning based. That is K-SVD and optimal direction method. A mathematical model represents only one SR fusion structure[2]. It causes poor representation for the original image. Hybrid mathematical based dictionary is the best choice for SR fusion structure but still lack of ability to represent various image types. Dictionaries are divided into sub-dictionaries and a sub-dictionary can be refined with training samples into more structural groups. This is the way of making sub-dictionary to produce a best structure. On the hole, a dictionary may have well representation.

\section{(c) Methods from other Domains}

Methods can be classified into two categories. One is from pixel domain and another one is transform domain.

Pixel Domain: The average weight of the input messages are considered as each pixel of the image fusion. Weights are determined based on different pixels activity[14]. For selecting highest activity pixels, support vector machines and neural networks are being used. Here wavelet coefficients are considered as features. Spatial context can be used by dividing images into uniform blocks. The spatial context can be improved with the help of each block. Quad-tree structures are used to get optimal subdivision block[15]. Region based image fusion methods also can be used irrespective of shapes. This is done by the image segmentation concept.
Next method is calculating the weights, and then optimizes it with the help of image matting. Edge-preserving filtering method is better than matting method. Random walker is another method and this method is improved as Maximum A Posteriori (MAP)[16]. These methods are fast and implementation wise ease. Optimal weights of different pixels are accurate assessment.

Transform Domain: Color space and dimension reduction based transforms are the other methods to apply image fusion. PCA (Principal Component Analysis), GS (Gram-Schmidt), HIS (Intensity-Hue-Saturation) transform are comes under transform domain[17]. These methods cause color distortions in the fused images. Linear regression model is used to handle color distortion problems. ICA (Independent Component Analysis) transform, intuitionistic fuzzy are used in image fusion.

\section{(d) Hybrid Transforms}

Low frequency components are not performed in multi-scale transform. Small-scale images are difficult in SR based fusion. IHS and PCA transform methods can be used only in remote sensing areas. So by grouping these methods can perform well than individual performance. That is decomposing images using multi-scale method, then applying SR for low frequency components and existing fusion rules are applied to high frequency components and finally inverse multi-scale transform is applied[18]. Similarly so many techniques are available by combining these methods according to the usage.

\section{Applications}

Fusion images are used in different areas especially in medical diagnosis. MRI (Magnetic Resonance Imaging) images, CT (Computerized Tomography) images and PET (Position Emission Tomography) images are some examples for using fusion techniques in medical diagnosis[19]. These images are used together to apply fusion techniques which increases accuracy. SPECT (Single Photon Emission Computed Tomography) and Ultra Sound images can also apply fusion techniques.

\section{Comparison of Various Fusion Schemes and Transforms Used in Various Approaches}

\begin{tabular}{|c|c|c|c|}
\hline S.No & Approaches & $\begin{array}{r}\text { Transforms } \\
\end{array}$ & Fusion Strategies \\
\hline 1 & Sparse Representation & $\begin{array}{l}\text { Group SR, Gradient Constrained SR, Orthogonal } \\
\text { matching Pursuit, SR with over -complete dictionary, } \\
\text { Simultaneous OMP, Spectral and spatial details } \\
\text { dictionary }\end{array}$ & $\begin{array}{l}\text { Choose-Max And Weighted Average Based } \\
\text { Coefficients Combining, Window Based Activity } \\
\text { Level Measurement, Spatial context based weighted } \\
\text { average }\end{array}$ \\
\hline 2 & $\begin{array}{l}\text { Multi-Scale } \\
\text { Decomposition }\end{array}$ & $\begin{array}{l}\text { Pyramid, Wavelet, Complex Wavelet, Shearlet, } \\
\text { Contourlet, Curvelet, Anisotropic heat diffusion, } \\
\text { edge-preserving decompositions, Optimal scale } \\
\text { transform and Support Value Transform. }\end{array}$ & $\begin{array}{l}\text { Window and Region based activity level } \\
\text { measurement, Coefficient, Choose-max, Weighted } \\
\text { Average, Optimization based method, Substitution } \\
\text { based, Cross scale fusion rule, Guided filtering } \\
\text { based weighted average }\end{array}$ \\
\hline 3 & Others & $\begin{array}{l}\text { PCA (Principal Component Analysis), GS (Gram- } \\
\text { Schmidt), Spatial Domain- Non Transform, } \\
\text { Independent Component Analysis, HIS (Intensity- } \\
\text { Hue-Saturation, Fuzzy theory and Gradient Domain }\end{array}$ & $\begin{array}{l}\text { Block and Region based, Machine learning, Spatial } \\
\text { Context based and Model based and Component } \\
\text { Substitution }\end{array}$ \\
\hline 4 & Hybrid & $\begin{array}{l}\text { Multi-scale transform-SR, Morphological component } \\
\text { analysis-SR, Hybrid wavelet -contourlet, HIS-Retina- } \\
\text { Inspired models, HIS-Wavelet }\end{array}$ & $\begin{array}{l}\text { Choose-max and weighted-average based, } \\
\text { Coefficient and window based, Component } \\
\text { substitution, integration of component substitution } \\
\text { and weighted average. }\end{array}$ \\
\hline
\end{tabular}

\section{Discussion}

Intensity-Hue Saturation method used to get high spatial resolution and it will not be decomposed into different frequencies. The Brovey Transform method overcomes the drawbacks of multiplicative method. The principal component analysis method creates uncorrelated images and it is an alternative method of panchromatic band. Empirical mode decomposition method decomposes into AM/FM modulated components called 'Intrinsic Mode Functions' and having constraints such as equal number of IMF from each source and must match for meaningful comparison. Discrete Wavelet Transform method can be applied only with higher spatial resolution images. 


\section{Conclusion}

In medical field, image fusion becomes a valuable technique to improve the quality of image in order to diagnose. Vision-related processing task that is edge detection, stereo matching and image segmentations are the parameters can give more accurate results. Image fusion is mainly depends up on the transformation of informative features from various image sources into fused images. In this article multiple images of a same scene are taken and each may contain over-saturated region or under-saturated region. So the selection of best illumination image at a particular pixel location and heterogeneous images are the extensions of image fusions in future and widely used.

\section{References}

[1] B.A. Olshausen , J.F. David , Emergence of simple-cell receptive field proper- ties by learning a sparse code for natural images, Nature 381 (6583) (1996) 607-609 .

[2] R. Rubinstein , A. Bruckstein , M. Elad, Dictionaries for sparse representation modeling, Proc. IEEE 98 (6) (2010) 1045-1057

[3] T. Mertens , J. Kautz , F.V. Reeth , Exposure fusion, in Proceedings of Pacific Conference on Computer Graphics and Applications, 2007, pp. 382-390

[4] T. Mertens , J. Kautz , F.V. Reeth , Exposure fusion, in Proceedings of Pacific Conference on Computer Graphics and Applications, 2007, pp. 382-390.

[5] A. Ben Hamza , Y. He , H. Krim , A. Willsky , A multiscale approach to pixel-level image fusion, Integrated Computer-Aided Engineering 12 (2) (2005) 135-146.

[6] J.J. Lewis , R.J.O. Callaghan , S.G. Nikolov , D.R. Bull , N Canagarajah, Pixel- and region-based image fusion with complex wavelets, Inf. Fus. 8 (2) (2007) 119-130

[7] F. Nencini , A. Garzelli, S. Baronti, L. Alparone, Remote sensing image fusion using the curvelet transform, Inf. Fus. 8 (2) (2007) 143-156. Special Issue on Image Fusion: Advances in the State of the Art

[8] T. Li , Y. Wang , Biological image fusion using a NSCT based variable-weight method, Inf. Fus. 12 (2) (2011) 85-92

[9] S. Yang , M. Wang , L. Jiao , R. Wu , Z. Wang, Image fusion based on a new con- tourlet packet, Inf. Fus. 11 (2) (2010) 78-84

[10] L. Yang , B. Guo , W. Ni , Multimodality medical image fusion based on mul- tiscale geometric analysis of contourlet transform Neurocomputing 72 (1-3) (2008) 203-211.

[11] K.P. Upla , M.V. Joshi , P.P. Gajjar , An edge preserving multiresolution fusion: use of contourlet transform and MRF prior, IEEE Trans. Geosci. Remote Sens- ing 53 (6) (2015) 3210-3220 .

[12] C. Chen , Y. Li , W. Liu , J. Huang , Image fusion with local spectral consistency and dynamic gradient sparsity, in: Proceedings of IEEE Conference on Com- puter Vision and Pattern Recognition, 2014, pp. 2760-2765

[13] Y.C. Pati , R. Rezaiifar , P.S. Krishnaprasad, Orthogonal matching pursuit: recur- sive function approximation with applications to wavelet decomposition, in: Proceedings of Asilomar Conference on Signals, Systems and Computers, vol. 1, 1993, pp. 40-44.

[14] N.Gangapure,S.Banerjee,A.S.Chowdhury, Steerable local frequency based multispectral multofocus image fusion,Inf.Fus.23(1)2015 (99-115)

[15] I.De,B.Chanda, Multi-focus image fusion using a morphologybased focus measure in a quad-tree structure,Inf.Fus.14(2)(2013) 136-146.

[16] R.Shen, I.Cheng,A.Basu,QoE-based multi-exposure fusion in hierarchy-cal multivariate Gaussian CRF, IEEE Trans.Image Process. 22(6) (2013) 2469-2478.

[17] H.R.Shahdoosti,H.Ghassemian,Combining the spectral PCA and spatial PCA fusion methods by an optimal filter, Inf Fus.(27)(1)(2016)150-160.

[18] Y.Liu,S.Liu,Z.Wang,A general framework for image fusion based on mutli-scale transform and sparse representation, Inf.Fus.24(1)(2015)147-164.

[19] L.Wang,B.Li,L.Tian,EGGDD:An explicit dependency model for multi-modal medical image fusion in shift-variant shearle transform domain,Inf.Fus.19(1)(2014)29-37. 